УДК 528.88; 53.082.56; 504.064

ФЛУОРЕСЦЕНТНЫЙ МЕТОД ИЗМЕРЕНИЯ ТОЛЩИНЫ НЕФТЯНОЙ ПЛЕНКИ НА ПОВЕРХНОСТИ МОРЯ

FLUORESCENT METHOD FOR MEASUREMENT OF OIL FILM THICKNESS ON THE SEA SURFACE

\title{
Н.3. Мурсалов
}

Государственный институт водных проблем, г. Баку, Азербайджанская Республика

\section{С.Н. Абдуллаева}

Азербайджанский государственный университет

нефти и промышленности, г. Баку, Азербайджанская Республика

Nemat Z. Mursalov

State Research Institute of Water Problems, Baku, Republic of Azerbaijan Sevindj N. Abdullayeva

Azerbaijan State University of Oil and Industry, Baku, Azerbaijan Republic e-mail: Nematmursalov@mail.ru

Аннотация. Статья посвящена разработке нового метода определения толщины нефтяной пленки на поверхности моря - флуоресцентного.

Загрязненность морской поверхности нефтью в настоящее время контролируется на трех уровнях: спутниковый контроль, контроль с использованием самолетов и других авиасредств, контроль с использованием морских судов и других плавсредств. В последнее два десятилетия с 
появлением лазерных средств дистанционного зондирования получило развитие третье направление. В сфере исследования загрязненности морской поверхности нефтью используется особый режим работы лидаров, в котором лазерный луч индуцирует флуоресценцию нефтяной пленки, при этом индуцированный флуоресцентный сигнал используется для анализа степени загрязненности поверхностных вод.

Авторами представлен анализ известных экспериментальных результатов исследований флуоресцентного сигнала, индуцированного лазерным излучателем при воздействии лазерного луча на нефтяную пленку на поверхности воды. Предложен метод определения толщины нефтяной пленки на поверхности воды по экстремуму интенсивности флуоресцентного излучения при изменении длины волны измеряемого сигнала. Составлен алгоритм реализации предлагаемого метода.

Предложенный метод и соответствующий алгоритм его реализации позволяют определить толщину пленки нефти известного типа при проведении измерений интенсивности флуоресцентного сигнала, индуцированного лазерным излучателем фиксированной длины волны по признаку достижения максимальной интенсивности флуоресцентного излучения при осуществлении пошагового изменения длины волны измеряемого флуоресцентного сигнала.

Abstract. The article is devoted to the development of a new method for determining the thickness of an oil film on the surface of the sea - fluorescent.

Oil pollution of the sea surface is currently monitored at three levels: satellite control, control using airplanes and other aircraft, control using sea vessels and other watercraft. In the last two decades, with the advent of laser remote sensing a third direction has been developed. In the field of studying sea surface pollution by oil, a special lidar regime is used, in which the laser beam induces fluorescence of 
the oil film, while the induced fluorescence signal is used to analyze the degree of pollution of surface waters.

The authors presented an analysis of the known experimental studies of the fluorescent signal induced by a laser emitter when a laser beam affects an oil film on a water surface. A method is proposed for determining the thickness of an oil film on a water surface by the fluorescent radiation intensity extremum when the wavelength of the measured signal changes. An algorithm for the implementation of the proposed method is compiled.

The proposed method and the corresponding algorithm for its implementation make it possible to determine the thickness of an oil film of a known type when measuring the intensity of a fluorescent signal induced by a laser emitter of a fixed wavelength on the basis of achieving the maximum intensity of fluorescent radiation when stepwise changing the wavelength of the measured fluorescent signal.

Ключевые слова: нефтяная пленка; флуоресцентное излучение; загрязнение морских вод; спектр; насыщение

Key words: oil film; fluorescence radiation; seawater pollution; spectrum; saturation

Загрязненность морской поверхности нефтью в настоящее время контролируется на трех уровнях: спутниковый контроль, контроль с использованием самолетов и других авиасредств, контроль с использованием морских судов и других плавсредств. В последнее два десятилетия с появлением лазерных средств дистанционного зондирования получило развитие третье направление [1-5]. В отличие от обычных лидаров, в сфере исследования загрязненности морской поверхности нефтью используется 
особый режим работы лидаров, в котором лазерный луч индуцирует флуоресценцию нефтяной пленки, при этом индуцированный флуоресцентный сигнал используется для анализа степени загрязненности поверхностных вод.

В общем случае лидар с лазерным индуцированием флуоресценции (LIF Lidar) состоит из собственно лазерного излучателя, спектрометра, телескопа приемного сигнала (рисунок 1).

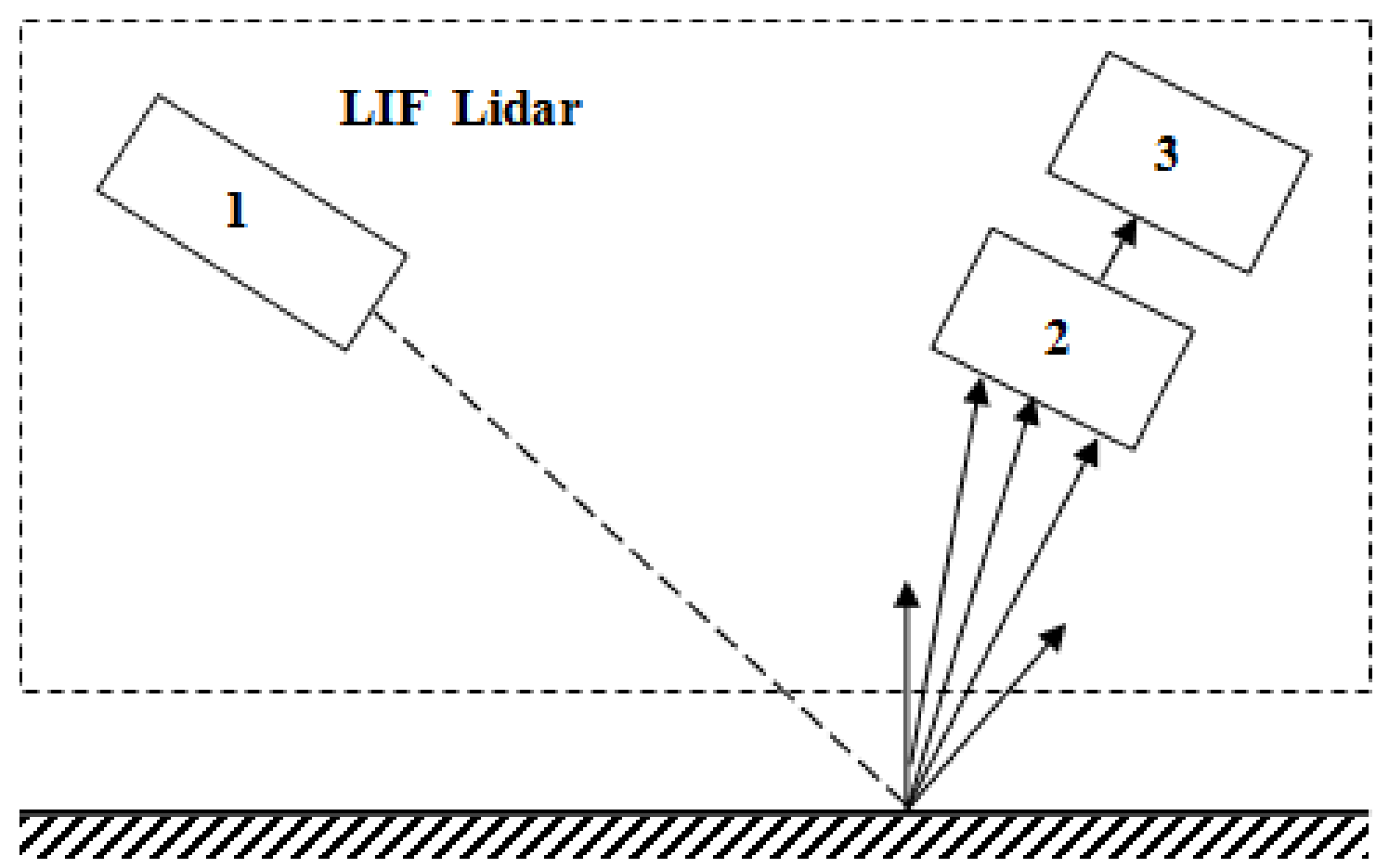

Морская поверхность

1 - лазерный излучатель;

2 - приемный телескоп;

3 - спектрометр

Рисунок 1. Блок-схема лидара с лазерным индуцированием флуоресценции 
В качестве примера исследования нефтяной пленки различных толщин можно привести результаты, полученные при изучении нефти типа «Azeri light» [6], лазерно-индуцированные флуоресцентные спектры которого приведены на рисунке 2.

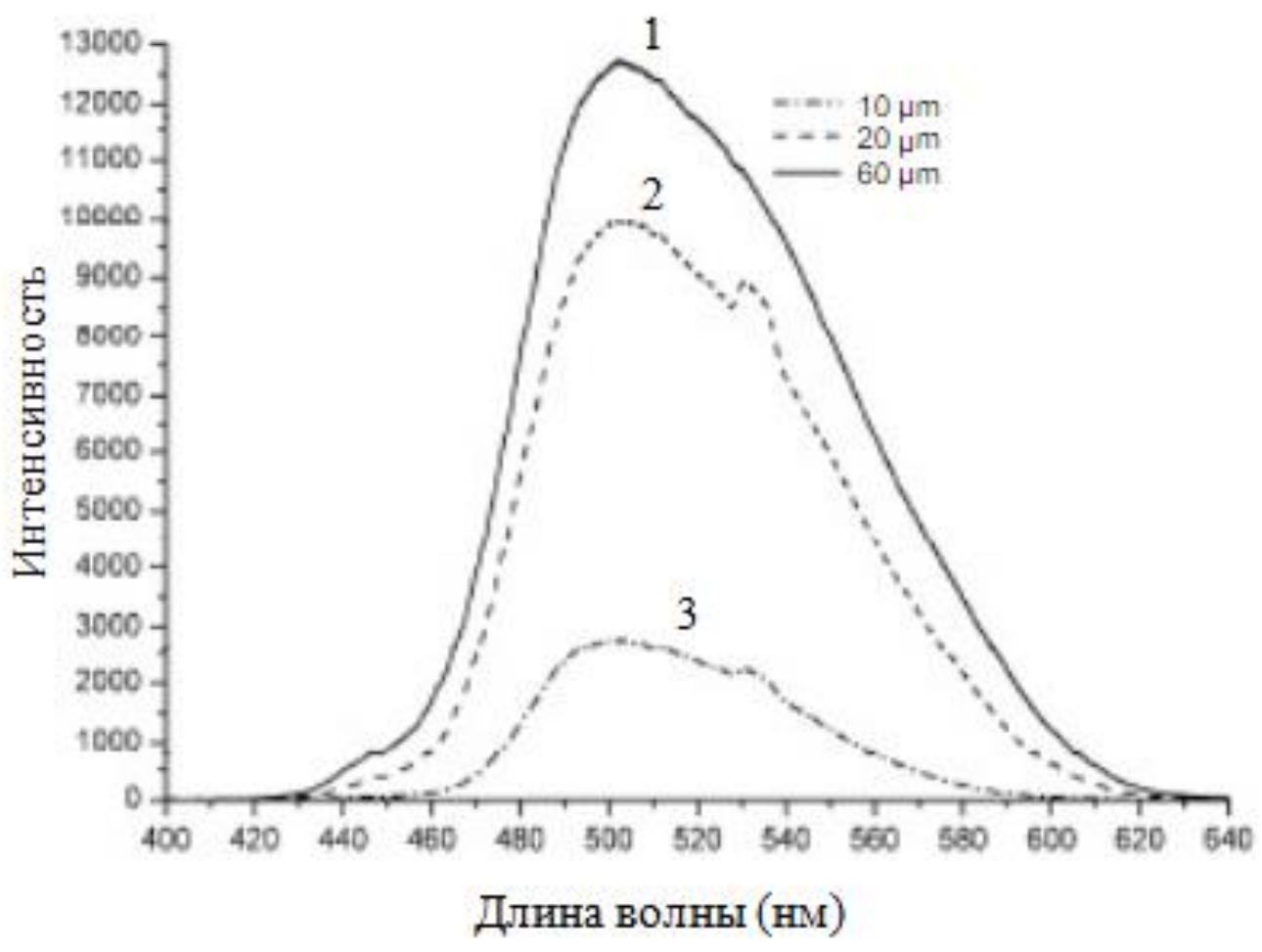

Толщина нефтяной пленки:

1 - 60 мкм; 2 - 20 мкм; 3 - 10 мкм

Рисунок 2. Лазерно-индуцированные флуоресцентные спектры нефти типа «Azeri light» при разных толщинах нефтяной пленки на поверхности воды [6]

Как видно из представленных на рисунке 2 графиков, спектр флуоресцентного сигнала нефти типа «Azeri light» имеет более широкополосный LIF спектр по сравнению с нефтью типа «Zafiro Blend», аналогичные кривые которого показаны на рисунке 3. 


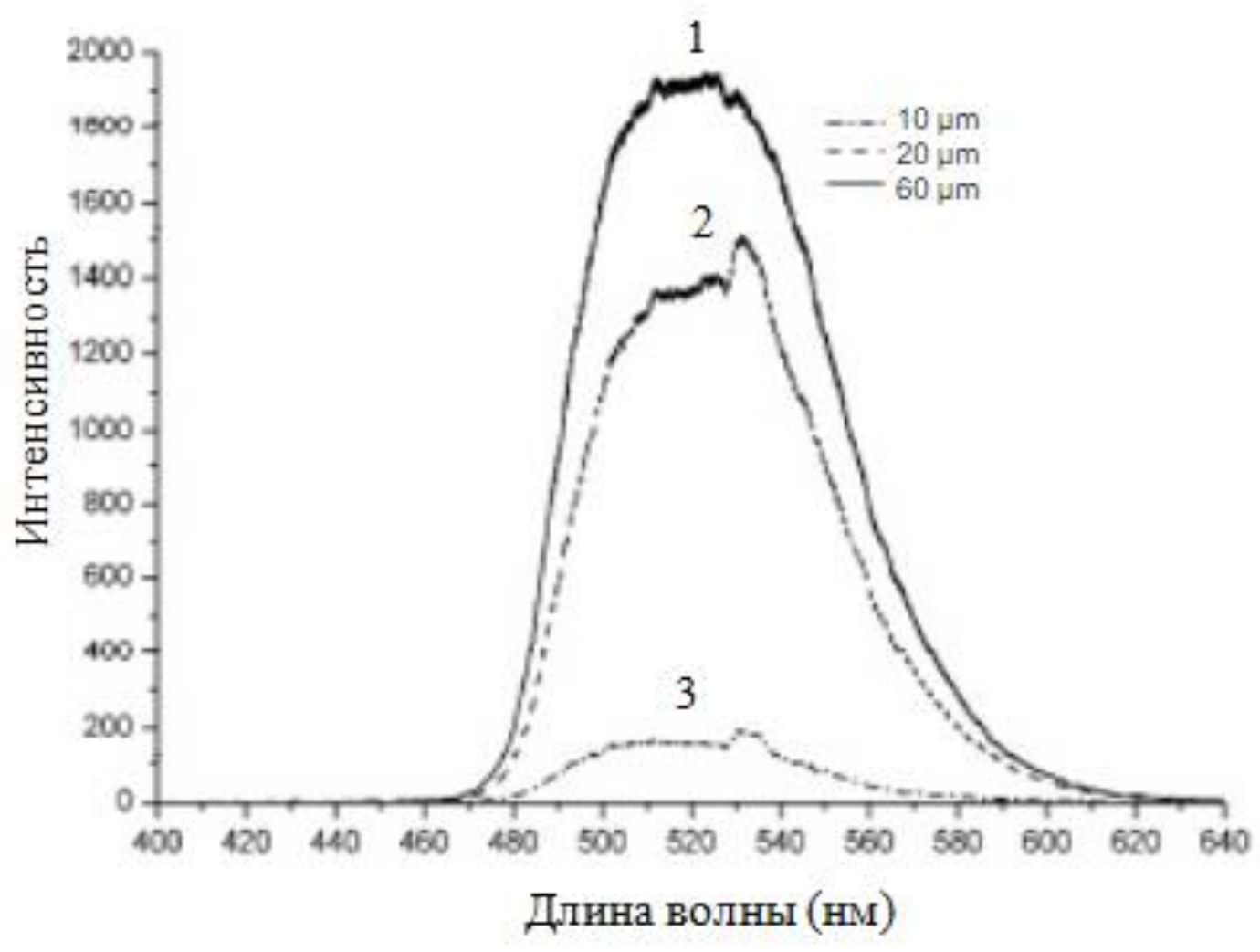

Толщина нефтяной пленки:

1 - 60 мкм; 2 - 20 мкм; 3 - 10 мкм

Рисунок 3. Лазерно-индуцированные флуоресцентные спектры нефти типа «Zafiro Blend» при разных толщинах нефтяной пленки на поверхности воды [6]

Как видно из графиков, показанных на рисунках 2 и 3, наблюдается увеличение интенсивности флуоресцентного сигнала с ростом толщины пленки в диапазоне 10-60 мкм.

Однако дальнейшее увеличение толщины нефтяной пленки сопровождается эффектом насыщения, что для рассмотренных типов нефти иллюстрируется на рисунке 4. 


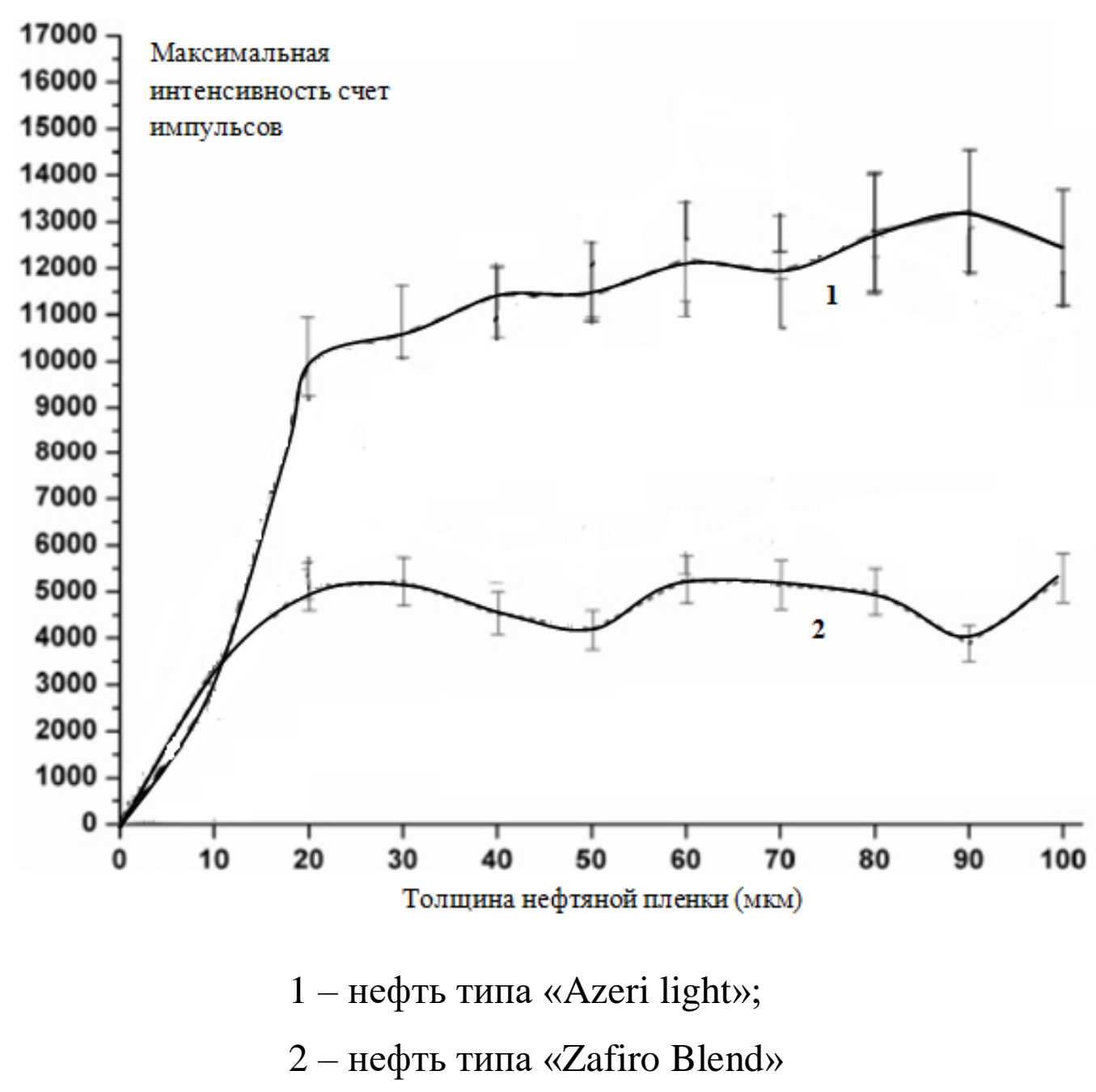

Рисунок 4. Иллюстрация эффекта насыщения для сравниваемых двух типов нефти при увеличении толщины пленки нефти в диапазоне 20-100 мкм

Таким образом, вышеприведенные данные показывают, что флуоресцентные сигналы, индуцированные лазером достаточно информативны и, в принципе, могут быть использованы для определения как толщины нефтяной пленки, так и типа исследуемой нефти.

Целью настоящей работы является разработка теоретических основ предлагаемого нового метода определения толщины нефтяной пленки на морской поверхности.

Для изложения предлагаемого метода воспользуемся некоторыми данными, приведенными в [6]. 
Согласно [6], при толщине нефтяной пленки $\mathrm{d}$ спектральная интенсивность, определяемая общим количеством эмитируемых фотонов в течении лазерного импульса с единицы площади в спектральном интервале $(\lambda-\Delta \lambda)-(\lambda+\Delta \lambda)$ может быть вычислена из выражения:

$$
d S(d)=C_{0}\left(1-\exp \left[-\alpha_{e}+\alpha_{\lambda}\right] d\right) d \lambda,
$$

где $\alpha_{e}-$ коэффициент затухания в нефти лазерного излучения фиксированной длины волны;

$\alpha_{\lambda}-$ коэффициент затухания в нефти флуоресцентного излучения при длине волны $\lambda$;

$$
d \text { - толщина пленки; }
$$

$$
\mathrm{C}_{0}=\frac{\mathrm{E}_{0} \cdot \sigma \cdot n \cdot g_{\lambda}}{\alpha_{e}+\alpha_{\lambda}} \cdot \frac{\tau}{\tau^{*}}
$$

где Е - общий поток фотонов лазерного импульса;

$\sigma$ - пеперечное сечение оптического поглощения возбуждающего лазерного излучения;

$n$ - средняя молекулярная концентрация нефти;

$g_{\lambda}-$ коэффициент формы флуоресцентного излучения в указанном спектральном диапазоне;

$\frac{\tau}{\tau^{*}}-$ отношение эфективной и радиационной времен жизни в состоянии возбуждения.

Рассмотрим возможность разработки метода определения толщины пленки нефти на поверхности воды при известном типе нефти.

Полагаем, что при выбранной длине волны лазерного излучателя показатель $\alpha_{\lambda}$ является известной величиной. 
Примем следующее обозначение:

$$
\alpha_{e}+\alpha_{\lambda}=x
$$

С учетом выражений (1) - (3) имеем

$$
\frac{\delta S(d)}{d \lambda}=\frac{A}{x}[1-\exp (-x \cdot d)]
$$

где

$$
\mathrm{A}=\frac{\mathrm{E}_{0} \cdot \sigma \cdot n \cdot g_{\lambda} \cdot \tau}{\tau^{*}}
$$

Вследствие отмеченного выше эффекта насыщения примем условие:

$$
x \cdot d \ll 1 .
$$

В этом случае экспоненциальная функция может быть представлена с точностью $1 \%$ (при $x \cdot d<0,1)$ в следующем виде:

$$
\exp (-x d)=1-x d+\frac{(x d)^{2}}{2}
$$

Исследуем выражение (4) на экстремум по переменной $x$. Имеем

$$
-\frac{\mathrm{A}}{x^{2}}[1-\exp (-x d)]+\frac{\mathrm{A} \cdot d}{x} \cdot \exp (-x d)=0
$$

или

$$
\frac{-[1-\exp (-x d)]}{x^{2}}=d \cdot \exp (-x d)
$$


С учетом выражений (7) и (9) получим

$$
\frac{\left[1-1-\frac{(x d)^{2}}{2 !}\right]}{x}=d \cdot\left(1-x d+\frac{(x d)^{2}}{2 !}\right)
$$

Из выражения (10) следует

$$
\frac{\left[d-\frac{(x d)^{2}}{2 !}\right]}{x}=1-x d+\frac{(x d)^{2}}{2 !}
$$

Из выражения (10) находим

$$
1-\frac{x d}{2 !}=1-x d+\frac{(x d)^{2}}{2 !}
$$

или

$$
d=\frac{1}{x}=\frac{1}{\alpha_{e}+\alpha_{\lambda}}
$$

Таким образом, при условии (13) выражение (4) достигает экстремума. Можно показать, что экстремум является максимумом.

На основании полученных результатов предлагается метод определения толщины нефтяной пленки, который алгоритмически может быть изложен в виде следующей последовательности проводимых операций.

Начало. Известны тип нефти и $\alpha_{\lambda}$; имеется аппаратура для измерения $\delta S(d)$ в диапазоне длин волн $\left(\lambda-\frac{\Delta \lambda}{2} ; \lambda+\frac{\Delta \lambda}{2}\right)$.

Осуществляется изменение волны $\lambda$ измерения $\delta S(d)$ что приводит к изменению показателя $\alpha_{e}$. 
При выполнении равенства (13) показатель $\delta S(d)$ достигает своего максимума, что регистрируется соответствующей измерительной аппаратурой.

$\mathrm{B}$ момент $t_{0}$, когда $\delta S(d)$ достигает максимума, изменение длины волны регистрируемого флуоресцентного сигнала прекращается, а величина $d$ определяется как

$$
d=\frac{1}{\alpha_{e}\left(t_{0}\right)+\alpha_{\lambda}}
$$

Блок-схема вышеизложенного алгоритма измерения показана на рисунке 5.

Таким образом, предлагаемый метод и соответствующий алгоритм его реализации позволяют определить толщину пленки нефти известного типа при проведении измерений интенсивности флуоресцентного сигнала, индуцированного лазерным излучателем фиксированной длины волны, по признаку достижения максимальной интенсивности флуоресцентного излучения при осуществлении пошагового изменения длины волны измеряемого флуоресцентного сигнала. 


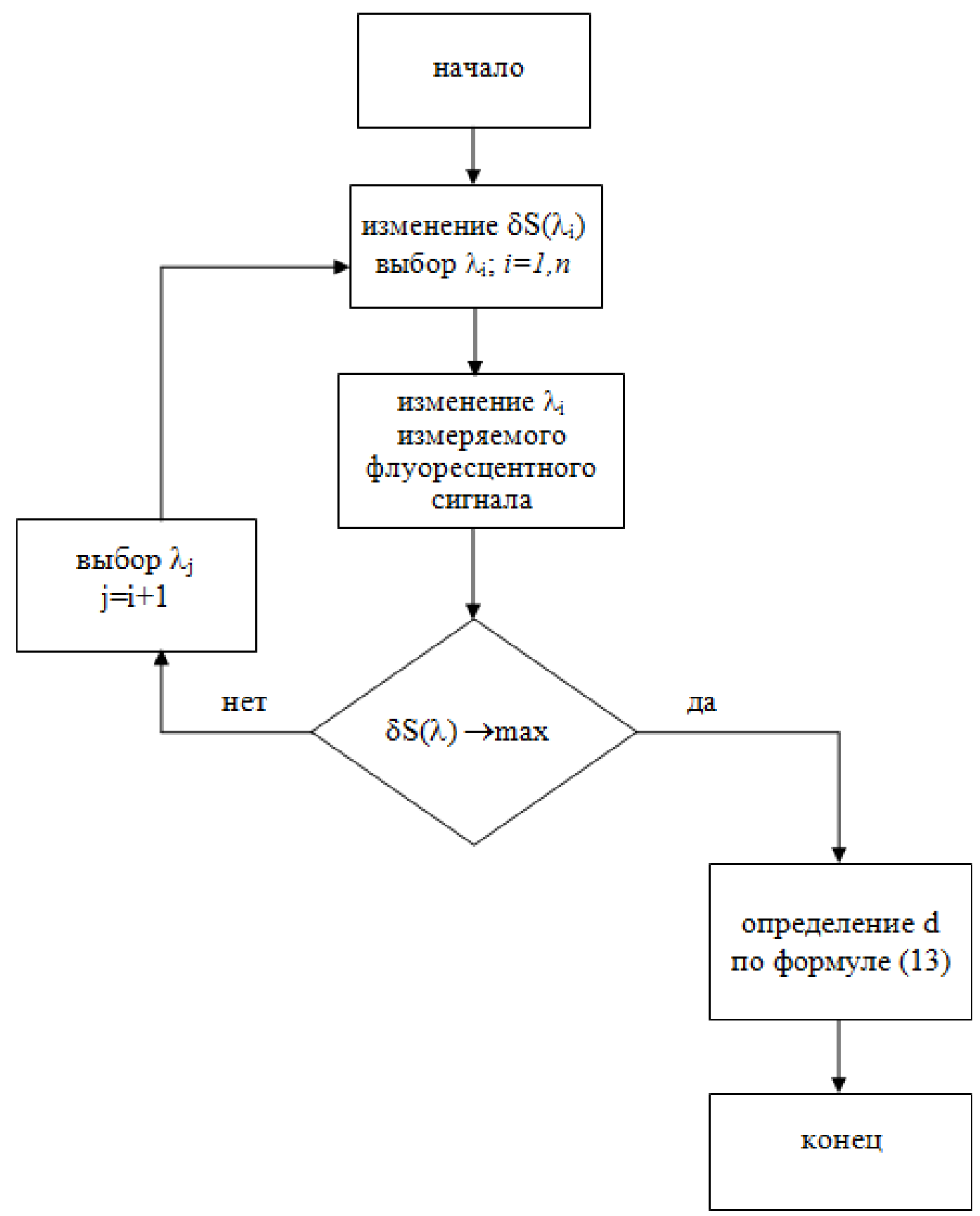

Рисунок 5. Блок-схема алгоритма измерения

\section{Выводы}

1. Представлен анализ известных экспериментальных результатов исследований флуоресцентного сигнала, индуцированного лазерным 
излучателем при воздействии лазерного луча на нефтяную пленку на поверхности воды.

2. Предложен метод определения толщины нефтяной пленки на поверхности воды по экстремуму интенсивности флуоресцентного излучения при изменении длины волны измеряемого сигнала.

3. Разработан алгоритм реализации предлагаемого метода.

\section{Список используемых источников}

1. Gasowski R., Mrozek-Lejman J. Fluorometric Method for Determining the Thickness of a Crude Oil Film Formed on the Water Surface // Oceanologia. 1994. No. 36 (2). P. 121-135.

2. MacHutchon K.R. Airborne Laser Induced Fluorescence as a Means for the Effective Management of Oil Spills, and for the Enforcement of Oil Discharge Regulations // WIT Transactions on the Built Environment. 2003. Vol. 70. P. 185194. DOI: $10.2495 / C E 030191$.

3. Hoge F.E., Swift R.N. Oil Film Thickness Measurement Using Airborne Laser - Induced Water Raman Backscatter // Applied Optics. 1980. Vol. 19. Issue 19. P. 3269-3281. DOI: 10.1364/AO.19.003269.

4. Ostroski G.M., Ghandhi J.B. Laser - Induced Fluorescence Measurement of the Oil Film Thickness in an Internal Combustion Engine // Optical Technology in Fluid, Thermal, and Combustion Flow III: Proceedings of SPIE 3172. San Diego, USA. 1997. Vol. 3172. URL: https://www.spiedigitallibrary.org/conferenceproceedings-of-spie/3172/1/Laser-induced-fluorescence-measurement-of-the-oilfilm-thickness-in/10.1117/12.279759.short?SSO=1 (дата обращения: 03.12.2019). DOI: $10.1117 / 12.279759$.

5. Hoge F.E. Oil Film Thickness Using Airborne Laser - Induced Oil Fluorescence Backscatter // Applied Optics. 1983. Vol. 22. Issue 21. P. 3316-3318. DOI: 10.1364/AO.22.003316. 
6. Utkin A.B., Lavrov A., Vilar R. Evaluation of Oil Spills by Laser Induced Fluorescence Spectra // International Conference on Lasers, Applications, and Technologies: Proceedings of SPIE 7994, LAT 2010. Kazan, Russian Federation. 2011. Vol. 7994. URL: https://www.academia.edu/32868848/Evaluation_ of_oil_spills_by_laser_induced_fluorescence_spectra_ (дата обращения: 04.12.2019). DOI: $10.1117 / 12.880750$.

\section{References}

1. Gasowski R., Mrozek-Lejman J. Fluorometric Method for Determining the Thickness of a Crude Oil Film Formed on the Water Surface. Oceanologia, 1994, No. 36 (2), pp. 121-135.

2. MacHutchon K.R. Airborne Laser Induced Fluorescence as a Means for the Effective Management of Oil Spills, and for the Enforcement of Oil Discharge Regulations. WIT Transactions on the Built Environment, 2003, Vol. 70, pp. 185194. DOI: $10.2495 / C E 030191$.

3. Hoge F.E., Swift R.N. Oil Film Thickness Measurement Using Airborne Laser - Induced Water Raman Backscatter. Applied Optics, 1980, Vol. 19, Issue 19, pp. 3269-3281. DOI: 10.1364/AO.19.003269.

4. Ostroski G.M., Ghandhi J.B. Laser - Induced Fluorescence Measurement of the Oil Film Thickness in an Internal Combustion Engine. Proceedings of SPIE 3172 «Optical Technology in Fluid, Thermal, and Combustion Flow III». San Diego, USA, 1997, Vol. 3172. Available at: https://www.spiedigitallibrary.org/ conference-proceedings-of-spie/3172/1/Laser-induced-fluorescence-measurementof-the-oil-film-thickness-in/10.1117/12.279759.short?SSO=1 (accessed 03.12.2019). DOI: $10.1117 / 12.279759$.

5. Hoge F.E. Oil Film Thickness Using Airborne Laser - Induced Oil Fluorescence Backscatter. Applied Optics, 1983, Vol. 22, Issue 21, pp. 3316-3318. DOI: 10.1364/AO.22.003316. 
6. Utkin A.B., Lavrov A., Vilar R. Evaluation of Oil Spills by Laser Induced Fluorescence Spectra. Proceedings of SPIE 7994, LAT 2010 «International Conference on Lasers, Applications, and Technologies». Kazan, Russian Federation, 2011, Vol. 7994. Available at: https://www.academia.edu/32868848/ Evaluation_of_oil_spills_by_laser_induced_fluorescence_spectra_ (accessed 04.12.2019). DOI: $10.1117 / 12.880750$.

\section{Сведения об авторах}

\section{About the authors}

Мурсалов Немат Закир оглы, докторант Государственного института водных проблем, г. Баку, Азербайджанская Республика

Mursalov Nemat Z., Doctorant of State Institute of Water Problems, Baku, Republic of Azerbaijan

e-mail: Nematmursalov@mail.ru

Абдуллаева Севиндж Новруз гызы, канд. техн. наук, доцент кафедры «Инженерное приборостроение», Азербайджанский государственный университет нефти и промышленности, г. Баку, Азербайджанская Республика

Sevindj N. Abdullayeva, Candidate of Engineering Sciences, Assistant Professor of Instrument Development Engineering Department, Azerbaijan State University of Oil and Industry, Baku, Republic of Azerbaijan

e-mail: abdullayevasn@ rambler.ru 